\title{
VALORACIÓN ECONÓMICA DEL RUIDO: UNA REVISIÓN ANALÍTICA DE ESTUDIOS*
}

\author{
Francisco Correa Restrepo** \\ Juan David Osorio Múnera*** \\ Bernardo Patiño Valencia**** \\ Recibido: septiembre 05 de 2010 \\ Aceptado: septiembre 30 de 2011
}

\section{RESUMEN}

El objetivo de este trabajo es establecer el estado del arte sobre la valoración económica del ruido a través de un recorrido por los diversos estudios realizados en el entorno mundial. Así, se explican los conceptos, medidas y los métodos usualmente utilizados en la valoración económica del ruido. Luego, mediante el método de análisis de contenido, se revisan los estudios desarrollados en Europa, Estados Unidos y Latinoamérica vinculados a las principales fuentes generadoras de ruido, a saber: tráfico de carreteras, de trenes y aeronaves. Finalmente, el estudio concluye que, a la luz de los diversos estudios analizados, los impactos más relevantes del ruido son la incidencia negativa sobre el precio viviendas y las molestias percibidas por los individuos, lo que genera una alta disposición pagar por hogar por la reducción de los niveles de ruido.

\section{PALABRAS CLAVE}

Valoración de impactos ambientales, ruido, externalidad negativa, disposición a pagar, economía del bienestar

\section{CLASIFICACIÓN JEL} Q51, Q53; D62.

\section{CONTENIDO}

Introducción; 1. El ruido como externalidad negativa; 2. Definición y medidas descriptivas; 3. Enfoques establecidos para la valoración económica del ruido; 4. Revisión analítica de los estudios de valoración económica del ruido; 5. Conclusiones; Bibliografía.

\footnotetext{
Este artículo de investigación es derivado del proyecto de investigación titulado "Valoración económica de los impactos ambientales asociados a la emision de ruido de las instalaciones de EPM Telecomunicaciones S. A. ESP", el cual fue desarrollado por el Grupo de investigación de Economía y Negocios Internacionales (GENI), Facultad de Ciencias Económicas y Administrativas, Universidad de Medellín, Categoría C Colciencias, ejecutado durante el periodo noviembre de 2009- junio de 2010 y tuvo el apoyo financiero de EPM Telecomunicaciones S. A. ESP.

* Economista y especialista en Evaluación Socioeconómica de Proyectos, Universidad de Antioquia, Medellín, Colombia. Magíster en Ciencias Económicas, Área de Economía de la Energía y los Recursos Naturales, Universidad Nacional de Colombia. Profesor asociado y Coordinador del Grupo de Investigación GENI, Facultad de Ciencias Económicas y Administrativas, Universidad de Medellín, Colombia. Apartado aéreo 1983, carrera 87 № 30-65 bloque 5 oficina 107, Medellín, Colombia, Tel: (574) 3405278, Correo electrónico: fcorrea@udem.edu.co.

*.* Economista Industrial, Universidad de Medellín, Colombia. Magíster en Economía del Medio Ambiente y los Recursos Naturales, Universidad de los Andes-Universidad de Maryland, Bogotá. Profesor auxiliar, miembro del Grupo de Investigación GENI, Facultad de Ciencias Económicas y Administrativas, Universidad de Medellín, Colombia. Apartado aéreo 1983, carrera 87 No 30-65 bloque 6 oficina 108-1, Medellín, Colombia, Tel: (574) 3405468, Correo electrónico: jdosorio@udem.edu.co.

*.** Economista, Universidad de Medellín. Profesor Catedrático, Facultad de Ciencias Económicas y Administrativas, Universidad de Medellín, Colombia. Asistente de Investigación, Grupo de Investigación GENI, Universidad de Medellín, Colombia. Apartado aéreo 1983, carrera 87 No 30-65, bloque 6 oficina 108 1, Medellín, Colombia. Tel: 3405468. Correo electrónico: bpatino@udem.edu.co.
} 


\section{ECONOMIC VALUATION OF NOISE: AN ANALYTICAL STUDY REVIEW}

\section{ABSTRACT}

The objective of this article is to establish the state of art of economic valuation of noise though a broad review of studies done around the world. With these, the concepts, measures and most used methods for economic valuation of noise are explained. Afterwards, the studies done in Europe, United States and Latin America are reviewed within the content analysis method concentrating on the main noise sources like; road, railroad and aircraft traffic. Finally the study concludes that under the light of the different studies, the most relevant impacts of noise are the negative incidence over the price of housing and the troubles perceived by individuals, what generates a high disposition to pay by household the reductions of high noise levels.

\section{KEY WORDS}

Valuation of environmental impacts, noise, negative externality, paying willingness, Wellbeing economics.

\section{JEL CLASSIFICATION}

Q51, Q53, D62

\section{CONTENT}

Introduction; 1. Noise as negative externality; 2. Definitions and descriptive measurements; 3. established scopes for economic valuation of noise; 4. Analytical review of the studies on economic valuation of noise; 5 . Conclusions; Bibliography.

\section{VALORAÇÃO ECONÔMICA DO BARULHO: UMA REVISÃO ANALÍTICA DE ESTUDOS}

O objetivo deste trabalho é estabelecer o estado da arte sobre a valoração econômica do barulho a traves de um recorrido pelos diversos estudos realizados no entorno mundial. Assim, explicam-se os conceitos, medidas e métodos usualmente usados na valoração econômica do barulho. Depois, mediante o método de analise de conteúdo, revisam-se os estudos feitos na Europa, os Estados Unidos e Latino América vinculados as principais fontes geradoras de barulho; O trafico nas estradas, trems e aeronaves. Finalmente, o estudo conclui que, à luz dos diversos estudos analisados, os impactos mais relevantes do ruído são a incidência negativa sobre o preço das vivendas e as moléstias percebidas pelos indivíduos, o que gera uma alta disposição de pagar por lar pela redução dos níveis de barulho.

\section{PALAVRAS-CHAVE}

Valoração de impactos ambientais, barulho, externalidade negativa, disposição a pagar, economia do bem-estar

\section{CLASSIFICAÇÃO JEL}

Q51, Q53; D62.

\section{CONTEÚDO}

Introdução; 1. O barulho como externalidade negativa; 2. Definição e medidas descritivas, 3. Enfoques estabelecidos para a valoração econômica do barulho; 4. Revisão analítica dos estúdios de valoração econômica do barulho; 5 . Conclusões; Bibliografia. 


\section{INTRODUCCIÓN}

El incremento de las actividades económicas propias de las ciudades en desarrollo trae consigo múltiples externalidades negativas a la sociedad. Una de estas externalidades es la llamada contaminación acústica o contaminación por ruido. Según Regecove y Rellerova (1995), se denomina ruido a un sonido no articulado, que en función de factores como su intensidad y frecuencia, puede causar diversas molestias y en algunos casos afectaciones a la salud. Esta externalidad es considerada como un contaminante ambiental de bajo costo de generación y cuyo valor económico presenta una difícil medición ya que está ampliamente influenciada por la percepción de las comunidades o individuos afectados ${ }^{1}$. Además, bajo el supuesto de que el silencio es un bien público puro que no presenta un precio de mercado, se evidencia una difícil medición del impacto del ruido sobre el bienestar de la sociedad.

Ahora bien, en la literatura económica se pueden encontrar diferentes estudios que, tanto de forma teórica como empírica, han tratado de analizar y monetizar las implicaciones de la contaminación por el ruido generado por diferentes fuentes, como lo son el ruido por tráfico vehicular, el tráfico aéreo y el tráfico ferroviario, entre otros, mediante la utilización de diferentes métodos entre los que se encuentra el método de precios hedónicos (PH), el método de valoración contingente (VC) y el método de transferencia de beneficios (TB).

Así, con la creciente importancia del ruido como externalidad negativa, su impacto en el bienestar social, y su consideración como problemática ambiental, el presente artículo tiene como objetivo central establecer el estado del arte en torno al tema de la valoración económica del ruido, a partir de los diferentes métodos de valoración económi-

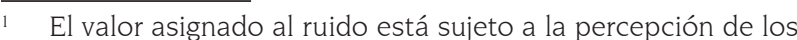
individuos afectados por este, condiciones culturales, sociales y de más factores, que generan apreciaciones diferentes en cada individuo, permitiendo variar el concepto de molestia relacionada a la consideración del ruido como externalidad negativa. ca aplicados en múltiples estudios desarrollados en países de la Unión Europea, Estados Unidos y América Latina.

De esta manera, el artículo consta de cinco secciones. La primera sección presenta la concepción del ruido como externalidad negativa, su efecto en el precio de las viviendas y los posibles efectos que se evidencian sobre la salud humana. La segunda plantea las diversas definiciones de ruido, así como las medidas descriptivas de ruido más utilizadas. En la tercera sección, se establecen los enfoques de valoración económica del ruido más utilizados. La cuarta sección presenta una revisión de los principales estudios de valoración económica del ruido realizados en el contexto internacional y latinoamericano. Por último, la quinta sección, expone las conclusiones del documento.

\section{EL RUIDO COMO EXTERNALIDAD NEGATIVA}

Uno de los problemas que han surgido a partir del crecimiento de las actividades económicas y, por supuesto, de las ciudades modernas es el ruido. A medida que crecen las ciudades, aumenta el ruido urbano. El ruido es considerado en la literatura como un contaminante de bajo costo de generación, que presenta características particulares que dificultan el establecimiento de su valor económico. En ese sentido, se plantean, como sus efectos más representativos, la incidencia sobre el precio de las viviendas y el efecto en la percepción humana mediante el sentido del oído.

Al considerar el efecto del ruido sobre el precio de las viviendas, los diferentes estudios permiten observar la relación inversa entre el incremento de los niveles de ruido y el precio de las viviendas y ,así, muestran, finalmente, una afectación negativa del ruido en el precio de éstas, así como disposición a pagar (DAP) positiva de los individuos por reducciones en los niveles de ruido (Flowerdew, 1972; Santford; Schultz y Green, 1988; Wilhelmsson, 2000; Strand y Vagnes, 2001; Bateman y otros, 2001; Marmolejo, 2008). 
Por otra parte, desde la consideración del ruido como agente afectador de la salud humana, estudios como el realizado por Martínez (2005, p.5), enuncian que la Organización Mundial de la Salud -OMS- ha estimado que en el mundo existen aproximadamente 120 millones de personas con dificultades auditivas invalidantes y que cerca de 500 millones de personas sufren por los altos niveles de ruido.

Asimismo, la OMS establece que 300 millones de personas en todo el mundo sienten incomodidad acústica, es decir, están expuestas a niveles sonoros entre 55 y $65 \mathrm{~dB}^{2}(\mathrm{~A})$. En este sentido, Martínez (2005) plantea que en los últimos 20 años, la OMS ha estudiado los efectos que provoca el ruido sobre la salud de las personas. La OMS (1999) establece que a un nivel de $30 \mathrm{~dB}$ no se puede conciliar el sueño. Este hecho disminuye la calidad del sueño. Ahora, en $40 \mathrm{~dB}$ se dificulta la comunicación verbal, para 75 dB se observa pérdida del oído a largo plazo y entre110-140 dB hay pérdida del oído a corto plazo, y por encima de $140 \mathrm{~dB}$ se presenta el umbral del dolor.

Bluhm, Nording y Berglin (2004) y Paz y Zannin (2008) han analizado el ruido por tráfico urbano y su correlación con el grado de molestia sobre el sueño de las personas, en especial los efectos que están relacionados con la salud y han encontrado una tasa de molestia por tráfico urbano equivalente al $76 \%$. Por su parte, Martínez (2005) plantea que el ruido por tráfico urbano aumenta el riesgo de infarto.

Ahora bien, esta conclusión no se puede generalizar de manera científica, pero desde el punto de vista de política ambiental sobre el control de la contaminación acústica, este riesgo estimado de vida es considerable y requiere ser reducido 3 . Por otro lado, un estudio sobre el impacto del ruido urbano

2 Unidad de medida de nivel sonoro con ponderación frecuencial (A).

3 La afectación a la salud no queda ahí. Martínez (2005) plantea que, adicional a los efectos sobre la audición humana, se han descrito, desde la literatura médica, otras afecciones en individuos expuestos a ruidos intensos, como son: hipertensión arterial, taquicardias, estrés, nerviosismo y disminución en el rendimiento físico. en los niños es el de Ising e Ising (2002). Según este estudio, los niveles de exposición al ruido se relacionan con el aumento de patologías como el asma, la bronquitis y la aparición de alergias, en períodos nocturnos en los cuales los niños están expuestos a altos niveles. Asimismo, estos autores encontraron que la regulación hormonal de los niños se altera al estar expuestos a altos niveles de ruido.

Después de considerar las características generales del ruido y las posibles afectaciones para la salud de las personas, a continuación, se consideran diferentes definiciones y conceptos técnicos sobre la medición del ruido.

\section{DEFINICIÓN Y MEDIDAS DESCRIPTIVAS}

En la literatura sobre ruido se consideran algunas definiciones. Estas se diferencian por su enfoque, que puede establecerse desde el punto de vista técnico, jurídico o de carácter social. En este sentido, Martínez (2005), desde un enfoque técnico, define el ruido como un fenómeno sonoro formado por vibraciones irregulares en frecuencia y amplitud por segundo, con distintos timbres, sujeto al material resonante que lo origina. Seguidamente, desde el punto de vista jurídico, el ruido se asocia a la definición expuesta por Ortega (2002), quien muestra que el ruido no sería considerado como tal, si no produjese un rechazo y efecto no deseado para quien lo percibe; tal es el caso del efecto sobre el sueño.

De otro lado, desde el punto de vista social, se considera el planteamiento de Lamarque (1975, p. 13) quien define el ruido como "un sonido o conjunto de sonidos desagradables o molestos". Por su parte, Sanz (1987, p. 23) considera que el ruido es "un sonido molesto e intempestivo que puede producir efectos fisiológicos y psicológicos no deseados en una persona o grupo de personas", mientras que López y Herranz (1991, p. 8) definen el ruido como "toda energía acústica susceptible de alterar el bienestar fisiológico o psicológico de las personas".

En esencia, cualquiera que sea la definición, técnica, jurídica o social, se habla del ruido como 
uno o diversos sonidos molestos que pueden generar efectos fisiológicos o psicológicos y sociales no deseados por las personas. Así, Martínez (2005), plantea que todo ruido pasa a ser ruido cuando se introduce la variable "molesto", pero incluso lo molesto se mueve en un entorno de subjetividad.

De otro lado, se puede considerar que el entorno en el cual se genera el ruido incide ampliamente en la afectación que este produce sobre poblaciones específicas. Por tal motivo, en el marco de una zona urbana, es posible clasificar diferentes entornos según el respectivo uso de suelos y las condiciones de calidad acústica, los cuales son: zonas de alta sensibilidad acústica, de moderada sensibilidad acústica, de baja sensibilidad acústica y zona de servidumbre. La zona de alta sensibilidad acústica comprende aquellas zonas en las cuales hay poblaciones muy susceptibles a presentar incomodidad por la percepción de ruido tales como zonas sanitarias (hospitales), las culturales (teatros), entre otros. Las zonas de moderada sensibilidad acústica son aquellos sectores que admiten una percepción del nivel sonoro intermedio, tales como las viviendas, hoteles o zonas de protección especial, como centros históricos.

Por su parte, las zonas de baja sensibilidad acústica comprenden aquellos sectores en los cuales no hay poblaciones sensibles a presentar molestias por ruido, lugares como restaurantes, bares y locales o centros comerciales. Por último, están las zonas de servidumbre, que son áreas en las cuales se experimentan o producen altos niveles de ruido tales como espacios cercanos a vías, ferrovías, u otros equipos públicos.

Finalmente, con respecto a la medición de ruido es posible describir los diversos indicadores utilizados para la medición de los niveles de ruido en el ambiente, catalogados como indicadores descriptivos de ruido. Entre estos indicadores, los más utilizados son: el índice de ruido urbano (IRU), el nivel equivalente día-noche, el nivel equivalente día-tarde-noche y el Índice de Depreciación de Ruido (NDI, en sus siglas en inglés).

El índice de ruido urbano (IRU), plantea que las molestias percibidas por el individuo están relacionadas con el nivel de ruido percibido por este. Dicho indicador se define como: ) + 30, donde TNI es Índice de ruido urbano, $\mathrm{L}_{10}$ se define como pico de ruido, $\mathrm{L}_{90}$ se conoce como ruido de fondo y la diferencia entre $\mathrm{L}_{10}-\mathrm{L}_{90}$ se conoce como el clima de ruido (Martínez, 2005) ${ }^{4}$.

Por su parte, el nivel equivalente día-noche se denomina $\mathrm{L}_{d n} \mathrm{y}$ busca dar una idea del nivel de ruido en las 24 horas del día. Para calcular este indicador se mide un nivel equivalente en un período de 16 horas al día $\left(L_{d}\right)$ y un nivel equivalente 8 horas en la noche $\left(\mathrm{L}_{\mathrm{n}}\right)$. A esta última medición se le suma $10 \mathrm{~dB}$, bajo la consideración que la población en la noche es más sensible al ruido5. Luego, se promedia con el $\mathrm{L}_{\mathrm{eq}}$ (A) del día.

Por otro lado, respecto al nivel equivalente día-tardenoche se puede decir que este indicador se tiene en cuenta ya que en algunos países se considera como hora sensible para la percepción de ruido el periodo denominado atardecer, como es el caso de España donde 3 a 5 pm hay posibilidades de hacer la "siesta".

Los indicadores de ruido, $\mathrm{L}_{\mathrm{dn}} \mathrm{y} \mathrm{L}_{\mathrm{d}}$, son utilizados con frecuencia en la literatura económica. De manera similar, el NDI se utiliza en estudios de valoración económica, para medir el impacto del ruido sobre el valor de propiedades y viviendas. NDI representa la reducción porcentual en el precio de los inmuebles por cada unidad de variación del sonido (Walters, 1975). Generalmente, este indicador es usado en valoraciones económicas de ruido que utilizan la metodología de precios hedónicos con el objetivo de evaluar el impacto del ruido sobre el valor de las viviendas cercanas a la fuente de ruido.

En la literatura se plantea que el IRU es el mejor indicador de impacto por niveles de ruido (Navrud, 2002; Botton, 2003).

5 Si bien, usualmente, los niveles sonoros disminuyen en este período (noche), su importancia relativa aumenta. 
Como conclusión preliminar, puede decirse que el ruido, como externalidad negativa, presenta dificultades para ser internalizada y, actualmente, es de gran interés público debido a la creciente contaminación acústica relacionada con el desproporcionado aumento de las actividades económicas en el entorno mundial. Esta situación ha llevado a los gobiernos a tomar medidas como la creación de mapas de ruido, especialmente de las zonas urbanas, para mejorar la información referente a la problemática ambiental con el objetivo de realizar acciones que busquen minimizar la afectación en el bienestar social.

En este sentido, se plantea que los métodos de valoración económica son de gran utilidad para apoyar acciones de política pública y planeación del territorio que vayan dirigidas a mitigar el impacto negativo de este problema ambiental. Así, mediante la utilización de métodos como precios hedónicos, valoración contingente y transferencia de beneficios se ha logrado generar información valiosa sobre el valor económico del ruido, la cual permite reducir la incertidumbre respecto a las posibles formas para ejercer un adecuado control de la contaminación auditiva y sus diferentes afectaciones a la sociedad. A continuación, se describirán los métodos utilizados para la valoración económica del ruido.

\section{ENFOOUES ESTABLECIDOS PARA LA VALORACIÓN ECONÓMICA DEL RUIDO}

Los estudios realizados sobre valoración económica del ruido se han centrado, principalmente, en establecer valores para las afectaciones, bajo el enfoque de la percepción de molestias asociadas a la exposición a diferentes niveles de ruido y el efecto que tiene sobre el valor de las viviendas. Así, los diferentes estudios encontrados en la literatura económica buscan establecer, principalmente, DAP por la disminución de los ruidos causados por diversas fuentes, entre las que se tienen como más comunes el ruido generado por tráfico vehicular, tráfico aéreo y transporte férreo.
En general, la utilización de las técnicas y métodos para desarrollar los ejercicios de valoración económica dependen de las preferencias establecidas (Lambert, Poisson y Champlovier, 2001). Así, en el marco del análisis de las preferencias, si se reconoce que estas son explícitas (declaradas), es posible recurrir al método de VC que basa la asignación de valor a las preferencias individuales establecidas a partir de respuestas directas de los consumidores (Haro y Taddei, 2010). De otro lado, si se parte de preferencias implícitas (reveladas), un método adecuado para la valoración económica del ruido sería el de los precios hedónicos, el cual analiza el bien ambiental mediante el establecimiento de una relación entre el precio de un bien mercadeable y los atributos ambientales relacionados con este (Garrod, y Willis, 1992).

La utilización de una u otra metodología depende de las características del ejercicio de valoración. Sin embargo, autores como Pearce y Turner (1990) recomiendan considerar inicialmente la utilización de métodos indirectos como el de precios hedónicos para la valoración ambiental ya que, al utilizar información disponible en los mercados, los resultados están menos sometidos a cuestionamientos, pues la información revela realmente las preferencias de los consumidores. No obstante, si no se dispone de información suficiente sobre el bien a valorar en los mercados, tal como se presenta con bienes ambientales con altos componentes de no uso, puede ser preferible la utilización del método de valoración contingente

Alternativamente, se ha utilizado el método de transferencia de beneficios como una herramienta que puede llegar a ser importante para la valoración económica del ruido. Dicho método toma información recogida en un estudio original para la aplicación de esta en un contexto diferente (Osorio, 2006). Este método es utilizado en caso tal de presentarse dificultades para aplicar una adecuada recolección de información primaria y se tienen los estudios y la documentación suficientes para realizar un ejercicio. 
Debe aclararse que, a pesar de que son escasas las aplicaciones de este método en torno a la valoración económica del ruido, puede tener un potencial de aplicación en situaciones donde no es posible, por restricciones de costo y tiempo, realizar un nuevo estudio de valoración económica en profundidad.

A continuación, se describen los métodos de valoración antes mencionados, los cuales son los frecuentemente utilizados en la valoración económica del ruido.

\subsection{El método de valoración contingente}

En el contexto de la externalidad por ruido, este método busca establecer la máxima DAP por la reducción en la contaminación del ruido. Alternativamente, con este método se puede establecer cuál es la mínima compensación que las personas están dispuestas a recibir por las afectaciones generadas por el ruido. Así, se concibe como un método de valoración directa de la pérdida (o ganancia) de bienestar debido al ruido por tráfico urbano, por ejemplo.

El Método VC se basa en información que es recolectada a través de encuestas. Su objetivo central es determinar los impactos sobre la sociedad que son generados por la disminución en la calidad de un bien ambiental (Uribe y Rueda, 2003). Para obtener una estimación del valor económico, en primer lugar se debe determinar cuál es el cambio en la calidad del bien ambiental (el nivel de reducción de ruido, por ejemplo) que se quiere valorar y cuál es la población afectada por este cambio. En segundo lugar, se utilizan encuestas para crear un mercado hipotético, en el cual se pregunta al individuo por la máxima disposición a pagar o la mínima disponibilidad a aceptar (DAA) por el cambio en el bien ambiental (por ejemplo, DAP por reducciones en niveles ruido, o DAA por sufrir incrementos en niveles de ruido). Con la información recolectada se realiza una estimación econométrica de la DAP o DAA media de la población y se estima el valor total asignado al impacto sobre el bienestar ${ }^{6}$.

Finalmente, puede decirse que el objetivo central al utilizar el método de valoración contingente para valorar a la externalidad generada por el ruido es hallar la disposición a pagar de los individuos por la reducción de un decibel (análisis marginal en términos de decibeles) en los niveles de ruido y, por lo tanto, calcular el valor total de la reducción de pasar de un nivel de ruido inicial (línea base) en un nivel especifico menor (nivel objetivo).

\subsection{El método de precios hedónicos}

El enfoque de los precios hedónicos es un método indirecto de valoración de activos ambientales que se basa en el valor de la propiedad (finca raíz) para estimar de manera indirecta el valor de atributos ambientales que influyen sobre el precio de dichos bienes. Cuando los individuos compran un bien mercadeable como la vivienda, también pagan por atributos de su entorno, como la calidad del aire, la cercanía a espacios naturales, el silencio, etc. Así, basado en esta realidad, el método de precios hedónicos permite valorar económicamente características no mercadeables como el silencio, cuando ellas están asociadas a bienes transables como una vivienda o una finca ${ }^{7}$.

Cuando se combina el paisaje, el aire limpio, el silencio u otros atributos de un bien, ellos pueden caracterizar bienes heterogéneos. En ciertas circunstancias, es posible separar los efectos de esos atributos sobre la utilidad que ellos generan. Así, resulta posible determinar cómo los cambios en los niveles de cada atributo afectan el precio del bien. Aunque, en general, no siempre se dispone de información que permita asignar un precio a los distintos atributos o características que componen determinados bienes, como por ejemplo la

6 Generalmente, las formas lineales o logarítmicas han sido las formas utilizadas para estimar econométricamente la DAP por reducciones de los niveles ruido.

El término hedónico nace del análisis de los precios y de la demanda por los distintos atributos de un bien, los cuales generan distintos niveles de placer (Caraballo y Mendieta, 2005). 
vivienda o la propiedad rural, es posible obtener observaciones sobre la cantidad de dinero que se paga por adquirirlos. Lo anterior permite valorar monetariamente atributos como la calidad ambiental o la presencia de parques o cuerpos de agua en la vecindad de bienes como la vivienda. Asimismo, este método permite determinar el impacto que podrían tener cambios de estos atributos sobre sus precios.

Para realizar una estimación del valor económico de la calidad ambiental a partir de la metodología de PH es necesario establecer la relación entre el precio de un bien mercadeable -vivienda- y los atributos ambientales relacionados con él. Un bien, como la vivienda, puede ser descrito por un conjunto de características estructurales. Las más comunes son el tamaño, número de habitaciones, número de baños, número de garajes, tipo de vivienda (casa o apartamento), edad, materiales. Adicionalmente, ese bien puede ser descrito por un conjunto de características del entorno. Estás características pueden dividirse en dos grandes grupos: características socioeconómicas y características ambientales y urbanísticas.

Entre las características socioeconómicas de la vivienda están: las condiciones de inseguridad del entorno, cercanía a hospitales, cercanía a escuelas, el nivel de impuestos de la localidad y el acceso a centro comerciales, entre otros. Entre las características ambientales están: la contaminación atmosférica de la zona, nivel de ruido existente y contaminación visual, entre otros. Finalmente, entre las características urbanísticas se tienen la cercanía a espacios naturales, ciclo rutas y parques, entre otras (Correa y Osorio, 2010).

Además, se plantea que la metodología puede ser aplicada para valorar económicamente el impacto del ruido por medio del efecto que este factor tiene sobre el precio de la vivienda. No obstante, la metodología presenta limitaciones ya que solo permite captar, a través de la posesión de la vivienda, el cambio en el bienestar de aquellas personas que son afectadas por cambios en la calidad ambiental y más importante aún, requiere de información de precios de la vivienda en el mercado, en el cual hay imperfecciones en la información y en muchos casos es imposible recolectar la información necesaria para asegurar una buena e insesgada aplicación del modelo. Otra desventaja en la aplicación del modelo es el elevado costo en que se debe incurrir para recopilar la información.

Finalmente, una de las grandes debilidades de este método, en términos del precio implícito del factor medioambiental, es la sensibilidad a las condiciones de modelación como a las del mercado de los hogares ${ }^{8}$. En conclusión, solo modelos de precios hedónicos, basados en datos de transacciones de mercado deben ser utilizados. Como ventaja puntual e identificable del uso de la metodología de precio hedónico, se puede comentar que el uso de información proveniente de los mercados realmente refleja las preferencias de las personas, lo que puede reducir los cuestionamientos en la presentación de los resultados y estimaciones de los estudios.

\subsection{Método de transferencia de beneficios}

Rosenberger y Loomis (2003) definen este método como "la adaptación de información obtenida desde una investigación original para la aplicación de ésta en un contexto diferente de estudio". Osorio (2006) plantea que el método de trasferencia de beneficios se divide, esencialmente, en dos clases principales: transferencia de valores fijos y transferencia de funciones de análisis de meta-regresión. A continuación, se presenta una breve descripción de estos tres enfoques.

\subsubsection{Transferencias de valores fijos}

En la práctica, en este enfoque, los beneficios totales del sitio en el cual se va a establecer la política se estiman a través de valores por unidad

Los precios implícitos son bastante sensibles a la especificación del modelo, a los modelos de estimación, a la forma funcional y a los niveles de información acerca de los niveles de ruido percibidos por las personas según su situación. 
promedio obtenidos de los datos de un sitio de estudio ${ }^{9}$, mediante el uso de las estadísticas de una investigación original a un sitio de política ${ }^{10}$ de forma directa (Osorio, 2006). En la literatura se consideran dos formas de hacer transferencia de valores: transferencia de puntos estimados y transferencia de medidas de tendencia central.

Es importante resaltar que la limitación central del método de transferencia de valores aplicado al problema del ruido radica en que algunas veces los individuos no valoran igual los cambios ocurridos en diferentes sitios. Lo anterior se debe a dos razones fundamentales: primero, las personas establecidas en el sitio de la política pueden ser diferentes a las personas del sitio de estudio, en términos de la percepción del ruido como una molestia, diferencias en niveles de ingreso, otras características socioeconómicas. Segundo, aun si las preferencias de los individuos en el sitio de la política y de estudio son iguales, las oportunidades para evitar el ruido pueden no serlo.

\subsubsection{Transferencia de funciones}

La transferencia de funciones se orienta de forma más técnica que la transferencia de valores, lo cual permite establecer análisis más robustos. Este método transfiere modelos estadísticos o funciones que requieren relaciones vectoriales entre datos recolectados en un sitio de estudio, aplicados a un sitio de política. Es decir, los modelos estimados provenientes desde un sitio de estudio se utilizan con datos de variables explicativas obtenidas en sitios de establecimiento de políticas para estimar el valor por unidad y el valor total en el sitio de política. Cabe anotar que, en la literatura, la transferencia de funciones es considerada mejor opción que la transferencia de valores fijos. Esto se debe al mejor manejo de las características del sitio de política

9 Sitio o lugar del cual se posee información y datos del valor económico de determinado bien o recurso ambiental derivada de estudios e investigaciones anteriores.

10 Sitio o lugar en donde se desea conocer el valor económico de un determinado bien o servicio ambiental basado en la información de un sitio ya estudiado. y, por lo tanto, arroja valores más ajustados al contexto, mientras que la transferencia de valores fijos es invariante frente a las diferencias entre el sitio de estudio y el sitio de política (Osorio, 2006).

\subsubsection{Transferencia de funciones por meta-regresión}

En el caso de la transferencia de funciones de análisis de meta-regresión o meta-análisis, en lugar de transferir la función de beneficios de un solo estudio, como se aplicaba en el método anterior, se pueden combinar resultados de valoraciones de varios estudios con el fin de estimar una función de beneficios común. El enfoque de meta-análisis se ha utilizado para sintetizar hallazgos investigativos y obtener unos resultados más robustos que permitan el desarrollo de estimaciones más confiables. Muchas veces los datos aplicados en esta metodología son tomados de estudios aplicativos de los métodos de costos de viaje, valoración contingente o precios hedónicos, de los cuales, en gran cantidad de casos, es imposible realizar la determinación de las funciones de beneficio requeridas, ya que en la mayoría de los meta análisis la información secundaria debe ser recogida en los sitios donde las demás metodologías de valoración omiten datos y variables sobre las características poblacionales.

Según Osorio (2006, p. 114), los valores que se obtienen a través de la transferencia de beneficios están sujetos a errores. Este autor plantea que:

Una medida de error puede darse si un bien en el sitio de estudio es diferente del sitio de interés de implementación de política, esto es reflejado por diferentes atributos o diferencias en la calidad y cantidad de suministro de servicios [entre sitios]. Las medidas de error también pueden ser introducidas si las poblaciones del sitio de estudio y del sitio de la política tienen diferentes características socioeconómicas, las cuales influyen en los valores individuales, o si son de diferente tamaño poblacional lo cual influirá en el valor agregado. 
Asimismo, Stanley (2005) argumenta que las divergencias entre los mercados asociados a los sitios de valoración y las formas funcionales de los modelos econométricos pueden generar medidas de error en la valoración económica.

\section{REVISIÓN ANALÍTICA DE LOS ESTUDIOS DE VALORACIÓN ECONÓMICA DEL RUIDO}

Luego de haber descrito los métodos más utilizados para la valoración económica del ruido, se procede a realizar una revisión exhaustiva de los estudios más relevantes encontrados en literatura económica. En primer lugar, se revisan los principales estudios e investigaciones encontradas con aplicaciones referentes, en general, a Europa y Estados Unidos. Por último, se establecerá una revisión analítica de los estudios encontrados con aplicaciones en América Latina y Colombia.

\subsection{Revisión de estudios de valoración económica del ruido en el ámbito internacional: Europa y Estados Unidos}

Los estudios realizados en Europa y Estados unidos evidencian la utilización de los métodos de precios hedónicos ${ }^{11}$, valoración contingente y transferencia de beneficios (meta-análisis). Entre tanto, las unidades de medida más comúnmente utilizadas en estos países para determinar afectación del ruido en propiedades es el Índice de Depreciación de Ruido. Ahora, en el caso del establecimiento de molestias son los indicadores nivel equivalente día-noche y nivel día-tarde-noche ${ }^{12}$.

A continuación se presenta la revisión de estudios de valoración económica del impacto por ruido para Europa y Estados Unidos, dicha revisión está dividida según sea la fuente generadora de ruido, a saber: tráfico por carreteras, por aeronaves y por trenes.

11 El método hedónico, cuyo supuesto de partida es que el precio de un bien es una función de las características o atributos ambientales de su entorno. Este concepto se aplica de forma más visible para propiedad raíz como viviendas.

12 Aunque hay estudios donde los valores son expresados en términos de exposición a los niveles de ruido por persona.

\subsubsection{Ruido de tráfico por carreteras}

Las aproximaciones metodológicas para estimar económicamente los valores del ruido son similares entre los estudios y entre países. Así, Bateman y otros (2000) hacen un ejercicio de precios hedónicos para mostrar la valoración económica del ruido generado por tráfico por carretera y su efecto en el precio de las viviendas. Este autor utilizó sistemas de información geográfica (SIG) para obtener mejores datos de la localización de las viviendas y generar análisis más robustos. El ejercicio de PH fue aplicado a la localidad de Glasglow (Escocia), basado en la recolección de información para una muestra de 3.500 propiedades. Así, se determinó que el precio de las propiedades en dicha ciudad se ve disminuido en un $0.20 \%$ por cada decibel incrementado en el ruido generado por el tráfico por carretera.

Para el estudio se asumió como ruido bajo el asociado a $54 \mathrm{~dB}(\mathrm{~A})$ mientras que un ruido alto se asoció a los $78 \mathrm{~dB}(\mathrm{~A})$. Las variables utilizadas en la construcción del modelo hedónico están agrupadas en cuatro categorías: estructurales (número de habitaciones de la vivienda), accesibilidad (proximidad a hospitales), vecindad (tasa de desempleo local) y medioambientales (ruido por tráfico vehicular). Bajo el análisis de estas variables se evaluó la correlación de la variable ruido con la variable valor de las viviendas.

Por su parte, Pommerehne (1988), Soguel (1994) y Vainio (1995) aplicaron la metodología de valoración contingente para obtener resultados que pudieran evaluar la coherencia de los resultados a partir de la metodología de los precios hedónicos. Así, Pommerehne (1988), en un estudio realizado en Suiza, obtuvo estimaciones bastante confiables, a partir de las metodologías antes mencionadas y determinó la DAP de los habitantes de la cuidad de Basel por una reducción en los niveles de contaminación por ruido. El resultado obtenido, con el método $\mathrm{PH}$, fue una disposición a pagar igual a 116.13 euros/mes, mientras que con la metodología de VC halló una DAP de 110.25 euros/mes. 
El trabajo de Soguel (1994), con una aplicación en un contexto similar al estudio anterior, arrojó resultados bastante coherentes. Así, en el contexto de Europa, este autor, mediante la utilización del método aplicado a un ejercicio para valorar el ruido generado por el tráfico por carretera en la localidad de Neuchatel (Suiza), estimó la DAP por reducciones de los niveles de ruido perceptibles en un 50\%. Este autor encontró una disposición a pagar entre 60 a 71 euros (precios de 2001) por decibel por hogar al año.

Sin embargo, Vainio (1995) con una aplicación similar, realizada en la ciudad de Helsinki (Finlandia), desarrolló un ejercicio de valoración económica bajo la metodología del VC bajo la premisa de eliminar la molestia por ruido; así, obtuvo a niveles de $55 \mathrm{~dB}$ una DAP de 72 euros. Con un entorno igual al anterior, aplicado para la misma ciudad, Vainio (2001) obtuvo una DAP de 81 euros por año. Navrud (2000) mediante un ejercicio de valoración contingente halló la disposición a pagar media para los habitantes de Oslo (Noruega), por la eliminación de las molestias por ruido generadas a partir del ruido de tráfico vehicular. Para la realización de esta estimación todos los hogares entrevistados fueron expuestos a niveles de ruido de $65 \mathrm{~dB}$ (A). El resultado fue que no se encontró diferencia alguna en las medidas de reducción de ruido y la DAP, es decir, lo que los habitantes valoran es la reducción total de los niveles de ruido y no las pequeñas reducciones de este. Así, la estimación de la DAP estuvo entre el rango de 165 y 275 euros por decibel al año.

Con relación a los ejercicios de valoración realizados en Europa, que aplicaron medidas de estimación de valoración económica del ruido por medio de valores expresados en términos de niveles de exposición a ruido por persona, se encontraron resultados diversos. Así, Marmolejo y Fizzera (2008, p. 27) estimaron para la ciudad de Barcelona (España) el valor que para las personas tendría una eventual reducción del ruido urbano. En la encuesta de valoración contingente, la reducción del ruido ofrecida en el escenario de valoración equivalía a pasar "del nivel de ruido en un día laboral durante una hora punta a otro nivel inferior como el que se experimenta en un día laboral a las 21:00 horas". Los resultados muestran que el $27 \%$ de las personas manifiestaron respuestas de rechazo, y el 73\% manifestó su disponibilidad a pagar por el silencio. La DAP media fue de 3,39 euros por persona por mes y el $24 \%$ de los encuestados había efectuado adecuaciones en sus viviendas para mitigar el ruido.

En contraste con la baja DAP por reducción del ruido del estudio anterior, Salazar (2004) presenta los resultados de un estudio donde aplica la metodología de valoración contingente para estimar la disposición a pagar de los habitantes de Valencia (España) afectados por el ruido de la autopista AP-7. De este modo, obtuvo una DAP que varía entre 92,4 y 127,5 euros por decibel al año, por una disminución en los niveles promedio de ruido.

A continuación, en la tabla 1, se muestran algunos estudios importantes de valoración de ruido por tráfico vehicular en países como Suiza, Finlandia, Estados Unidos, Noruega, Francia y España.

Tabla 1. Principales estudios de valoración de ruido por tráfico vehicular

\begin{tabular}{|c|c|c|}
\hline $\begin{array}{c}\text { Estudio } \\
\text { (Metodología de } \\
\text { valoración) }\end{array}$ & $\begin{array}{l}\text { Sitio/ Descripción } \\
\text { del escenario } \\
\text { (Escenario de valo- } \\
\text { ración propuesto) }\end{array}$ & Resultados \\
\hline $\begin{array}{l}\text { Pom m e reh ne } \\
(1988) \\
\text { VC }\end{array}$ & $\begin{array}{l}\text { Suiza } \\
\text { (Reducción de } \\
50 \% \text { en los nive- } \\
\text { les de ruido) }\end{array}$ & DAP: 99€/dB/año \\
\hline $\begin{array}{l}\text { Soguel (1994) } \\
\text { VC }\end{array}$ & $\begin{array}{l}\text { Suiza } \\
\text { (Reducción de } \\
50 \% \text { en los nive- } \\
\text { les de ruido) }\end{array}$ & $\begin{array}{l}\text { DAP: } 60-71 € / \\
\text { dB/año }\end{array}$ \\
\hline $\begin{array}{l}\text { Vainio (1995) } \\
\text { VC }\end{array}$ & $\begin{array}{l}\text { Finlandia } \\
\text { (Eliminación de } \\
\text { las molestias por } \\
\text { ruido) }\end{array}$ & $\begin{array}{l}\text { DAP: } 72 € / d B / \\
\text { año. }\end{array}$ \\
\hline $\begin{array}{l}\text { Navrud (2000) } \\
\text { VC }\end{array}$ & $\begin{array}{l}\text { Noruega } \\
\text { (Exposición a } \\
\text { niveles de ruido } \\
\text { mayores a } 55 \mathrm{~dB} \text { ) }\end{array}$ & $\begin{array}{l}\text { DAP: } 165-275 € / \\
\text { dB/año }\end{array}$ \\
\hline
\end{tabular}


(continuación Tabla 1)

\begin{tabular}{|c|c|c|}
\hline $\begin{array}{c}\text { Estudio } \\
\text { (Metodología de } \\
\text { valoración) }\end{array}$ & $\begin{array}{l}\text { Sitio/ Descripción } \\
\text { del escenario } \\
\text { (Escenario de valo- } \\
\text { ración propuesto) }\end{array}$ & Resultados \\
\hline $\begin{array}{l}\text { Lambert y Cham- } \\
\text { plovier (2001). VC }\end{array}$ & $\begin{array}{l}\text { Francia } \\
\text { (Eliminación de } \\
\text { las molestias por } \\
\text { ruido) }\end{array}$ & 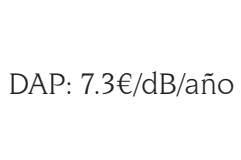 \\
\hline $\begin{array}{l}\text { Salazar (2004) } \\
\text { VC }\end{array}$ & $\begin{array}{l}\text { España } \\
\text { (Disminución de } \\
\text { los niveles de } \\
\text { ruido) }\end{array}$ & $\begin{array}{l}\text { D A P : } 92.4- \\
\text { 127.5€/dB/año }\end{array}$ \\
\hline $\begin{array}{l}\text { W i h e l m s s o n } \\
(2000) \\
\text { PH }\end{array}$ & $\begin{array}{l}\text { Estados unidos } \\
\text { (Afectación de } \\
\text { los niveles de } \\
\text { ruido) }\end{array}$ & $\begin{array}{l}\text { Reducción del } \\
\text { precio de las vi- } \\
\text { viendas en } 30 \%\end{array}$ \\
\hline
\end{tabular}

Fuente: Correa y Osorio (2010, p. 25)

Los anteriores estudios permiten establecer que se mantiene presente la relación positiva entre la DAP revelada por las personas y el incremento de la molestia generada por el ruido. Sin embargo, es necesario tener en cuenta las condiciones metodológicas presentes en cada estudio para analizar los resultados, ya que las estimaciones generadas mediante aplicaciones contingentes suelen ser inferiores a las estimaciones obtenidas por medio de ejercicios de precios hedónicos. Además, se debe aclarar que para los diferentes estudios de valoración contingente, se observan diferencias en la presentación de los escenarios.

Aunque todos los estudios relacionan la DAP preguntada con la reducción de los niveles de ruido, en algunos casos lo hacen sin especificar cuántos decibeles de ruido se van a reducir ${ }^{13}$. Estas condiciones en los escenarios pueden ser una característica relevante en la determinación del valor económico del ruido. Finalmente, se puede notar que para la valoración de ruido por tráfico por carretera, se utiliza más frecuentemente el método de valoración contingente.

13 En otros casos, se pregunta en términos de la disminución de la molestia.

\subsubsection{Ruido por aeronaves}

Al igual que en los estudios anteriores, existe una serie resultados diferentes del indicador NDI para cada sitio de política. Teóricamente, las aplicaciones metodológicas en diferentes ciudades, para diferentes años, deben tener resultados similares. Así, Schipper; Nijkamp y Rietveld (1998) determinaron que el precio implícito producto de las externalidades generadas por el ruido de los aviones es influenciado por un número de factores nunca antes tenidos en cuenta, tales como las fechas de ocurrencia de los impactos ambientales, los países donde se den y el grado de especificación con el cual se desarrollan estos estudios. Para esto, estos autores emplearon la metodología denominada meta-análisis en la que se realizó una aplicación guiada a determinar por qué varían los efectos del ruido producido por aeropuertos europeos con condiciones similares, de un estudio a otro. El resultado del estudio de transferencia de beneficios se aplica en la ciudad de Amsterdam (Holanda) como sitio de política. Para este ejercicio se utilizó como indicador principal de la relación de afectación del ruido sobre el valor de las propiedades el Índice Depreciación de Ruido. El estudio encontró que la depreciación de la propiedad debido al ruido es más alta para lugares en los que el precio promedio de la casa es más alto, lo cual confirma la hipótesis de que los bienes ambientales como la paz y la tranquilidad son bienes de lujo.

La investigación de Schipper; Nijkamp y Rietveld (1998) concluye que la línea base de partida para la determinación general del indicador NDI es de 0.33\% de depreciación del valor de las propiedades para países europeos, mientras que para Estados Unidos debería ser de 0.65\%. Similarmente, Bateman y otros (2001), por medio de la metodología de precios hedónicos, valoraron el impacto por ruido de los aviones. El resultado obtenido fue un NDI equivalente a $0.25 \%$, para las propiedades de Glasgow (Escocia). 
Miedema y Vos (1998), hicieron un ejercicio de meta-análisis para la ciudad de Leiden (Holanda) y utilizaron datos generados mediante el indicador de nivel de ruido día-noche. Este estudio consideró el ruido generado por tres fuentes: tráfico vehicular, tráfico de trenes y aeronaves, bajo la construcción de curvas de comportamiento que relacionan niveles de molestias (altas, medias y bajas) y niveles de ruido que oscilan entre 45 a $75 \mathrm{~dB}$ hora. El estudio en mención determinó una relación directa y creciente entre el incremento de los niveles de ruido percibidos y la molestia generada. Finalmente, se estableció que se asocian niveles de molestia mayores con el ruido generado por aeronaves que el generado por tráfico vehicular y férreo.

Por su parte, Meister y Donatelle (2000) evalúan el impacto de la aviación comercial en la salud humana mediante un ejercicio de valoración contingente en la Minneapolis/Saint Paul (Minnesota/ EEUU), para lo cual determinaron una muestra de individuos caracterizada por personas expuestas a altos y frecuentes niveles de ruido. Cerca de 2.000 personas fueron encuestadas y se buscó determinar: niveles de salud general, salud mental, vitalidad, niveles de estrés, molestias por ruido y niveles de sensibilidad al ruido. Mediante la relación de exposición-efecto, se determinó la existencia de una correlación entre el incremento de la percepción de molestia y el incremento de los niveles de ruido por encima de $55 \mathrm{~dB}(\mathrm{~A})$. Así, se logró comprobar que aquellos individuos con los peores cuadros de salud corresponden a las personas que experimentan con mayor severidad el ruido generado por los aviones.

En la temática de la valoración económica del ruido generado por aeronaves se han desarrollado algunos estudios de preferencias reveladas, pero son pocos los que presentan resultados relevantes sobre las estimaciones de las medidas de bienestar (DAP o DAA). Uno de los primeros estudios realizados fue conducido por Pommerehne (1988) para Suiza, en el cual se estableció un paralelo entre la valoración contingente y la metodología de precios hedónicos. De este proceso se obtuvo un rango estimado de la disposición a pagar por hogar, mensual, entre 32,34 y 47,04 euros, tenido una DAP media de 43 euros por decibel al año, respectivamente. Más tarde Thune-Larsen (1995), llevaron a cabo una valoración económica para el aeropuerto de Oslo (Noruega), en la cual se aplicaron de manera simultánea la metodología de VC y el análisis Conjoint ${ }^{14}$.

Por su parte, el estudio de Carlsson, Lampi y Martinsson (2004), analiza la propensión marginal a pagar por cambios en los niveles de ruido en relación con los cambios en los volúmenes de vuelos, para el aeropuerto de la ciudad de Estocolmo (Suecia) mediante un experimento de elección (Choice experiments ${ }^{15}$ ). De este modo, logra estimar las variaciones presentadas en la DAP para diferentes momentos del día y días de la semana. Una conclusión importante de este estudio es que los valores marginales de disponibilidad a pagar por cambios en los niveles de ruido son más altos para las dimensiones temporales mañana y tarde.

Ahora, Van Praag y Baarsma (2001) basaron su investigación en el supuesto de que los efectos de las externalidades se reflejan en las diferencias de los precios de las viviendas. Dado que los mercados de bienes raíz no son perfectos, se desarrolló un método de corrección que permitiera evaluar los daños en los hogares. Los autores evaluaron el valor monetario de los daños del ruido (molestias causadas por ruido de los aviones alrededor del

14 El análisis Conjoint, busca determinar el peso de los atributos que componen un determinado bien sobre el valor económico del mismo. Además, busca medir las preferencias por conjunto de esos atributos (Uribe y Rueda, 2003). Así, supone que el valor de un bien ambiental está constituido por el valor de sus atributos.

15 Los Choice Experiments o experimentos de elección, son metodologías en las cuales se les brinda a los encuestados un escenario hipotético compuesto por un conjunto de elecciones posibles, dentro de los cuales se les pide elegir sus preferidas. Así, cuando las personas realizan su elección implícitamente revelan el equilibrio entre los niveles de atributos de cada opción. 
aeropuerto de Ámsterdam) y el costo total del ruido determinado como la suma de las diferencias de precios hedónicos y un componente de costo residual. En el proceso metodológico, los costos residuales fueron evaluados a través de una encuesta, en la cual se incluía una escala ordinal de satisfacción que servía de medio para plasmar las respuestas individuales más notorias. El régimen de compensación planteado dependió, entre otras cosas, del nivel de ruido objetivo, los ingresos, los precios de cuenta de las diferencias de ruido y la presencia de aislamiento de ruido.

Por otra parte, Faburel (2001), hizo un estudio de valoración contingente, en Francia, cuyo objetivo era estimar los beneficios obtenidos a partir de la eliminación de las molestias por ruido de aeronaves, a través de la aplicación de una nueva política aérea que buscaba modificar los caminos de vuelo de los aviones. Para las zonas con mayores niveles de exposición la DAP anual por persona fue de 83 euros. Mientras que para zonas en las cuales existían menores niveles de exposición la DAP estimada fue de 11 euros.

De otro lado, Jasper y Straaten (2008), elaboraron un estudio por medio de la metodología de PH donde determinaron el precio de las viviendas con el fin de poder cuantificar los costos sociales de la perturbación por ruido producto de la externalidad negativa generada por las aeronaves. El estudio determina que el tráfico aéreo tiene el mayor impacto sobre los precios de las viviendas, seguido por el tráfico ferroviario y por el tráfico por carretera. Finalmente, estima que el beneficio marginal de la reducción por cada $\mathrm{dB}$ adicional es de 1.459 euros por vivienda al año.

La tabla 2 presenta el resumen de los resultados de los principales estudios revisados de valoración económica de impacto por ruido por aeronaves, relacionados con el escenario de valoración presentado.
Tabla 2. Principales estudios revisados de valoración económica de impacto por ruido por aeronaves

\begin{tabular}{|c|c|c|}
\hline $\begin{array}{c}\text { Estudio } \\
\text { (Metodología de } \\
\text { valoración) }\end{array}$ & $\begin{array}{l}\text { Sitio/ Des- } \\
\text { cripción del } \\
\text { escenario } \\
\text { (Escenario } \\
\text { de valoración } \\
\text { propuesto) }\end{array}$ & Resultados \\
\hline $\begin{array}{l}\text { Pommerehne } \\
\text { (1988) } \\
\text { VC }\end{array}$ & $\begin{array}{l}\text { Suiza } \\
\text { (Reducción de } \\
50 \% \text { en los ni- } \\
\text { veles de ruido) }\end{array}$ & $\begin{array}{l}\text { D.A.P: } 43 € / d B / \\
\text { año }\end{array}$ \\
\hline $\begin{array}{l}\text { Thune-Larsen } \\
\text { (1995) } \\
\text { VC y AC }\end{array}$ & $\begin{array}{l}\text { Noruega } \\
\text { (Reducción de } \\
50 \% \text { en los ni- } \\
\text { veles de ruido) }\end{array}$ & $\begin{array}{l}\text { D.A.P: } 190- \\
959 € / d B / a \tilde{o}\end{array}$ \\
\hline $\begin{array}{l}\text { Faburel (2001) } \\
\text { VC }\end{array}$ & $\begin{array}{l}\text { Francia } \\
\text { (Eliminación } \\
\text { de las moles- } \\
\text { tias por ruido) }\end{array}$ & $\begin{array}{l}8 € / \text { año para in- } \\
\text { crementos de } 10 \\
\text { dB. }\end{array}$ \\
\hline $\begin{array}{l}\text { Jasper y Straaten } \\
(2008) \\
\mathrm{PH}\end{array}$ & $\begin{array}{l}\text { Holanda } \\
\text { (Reducción de } \\
\text { los niveles de } \\
\text { ruido en área } \\
\text { de influencia) }\end{array}$ & $\begin{array}{l}\text { Beneficios margi } \\
\text { nales por hogar } \\
1.459 € / d B / a n ̃ o\end{array}$ \\
\hline
\end{tabular}

Fuente: Correa y Osorio (2010, p. 28)

Se evidencia en este tipo de estudios la preferencia de los investigadores por la utilización del método de precios hedónicos, tanto para los países europeos como para Estados Unidos. De otro lado, se concluye que el ruido afecta negativamente el precio de las propiedades. Finalmente, se plantea que el ruido de fondo experimentado en los lugares donde se estudia la valoración debe ser tenido en cuenta en el análisis con el fin de evitar la sobre estimación de la influencia de una fuente específica de ruido en las propiedades de las viviendas o en la salud humana.

\subsubsection{Ruido por trenes}

La mayoría de los estudios europeos referentes a la valoración de impactos ambientales de infraestructura ferroviaria aplican el análisis costobeneficio. Dentro de este de tipo de análisis se 
intenta monetizar el mayor número de impactos relevantes posibles. Sin embargo, es importante aclarar que existe una serie de directrices distintas para la valoración económica del ruido en la mayoría de los países occidentales y del norte de Europa. Además, en la mayoría de los estudios de valoración no se estiman todos los efectos del ruido, sino que se acostumbra tener en cuenta sólo las molestias del ruido en un contexto residencial.

Entre los estudios encontrados sobresale el trabajo de Strand y Vagnes (2001), quienes aplican la metodología de precios hedónicos para determinar el valor del ruido generado por los trenes en Noruega. El estudio determinó, mediante la utilización de la distancia de los hogares a las vías del tren, una disposición a pagar de $2.000 \mathrm{NOK}^{16}$, por metro de lejanía hacia las vías.

Por otro lado, mediante la utilización de la metodología de precios hedónicos, el estudio de Poon (1978) determina los costos económicos generados por el ruido de los trenes para la ciudad de Londres, mediante la determinación del cambio en el valor de las propiedades. Según los resultados establecidos por este estudio, es posible afirmar que las externalidades producto del funcionamiento de los trenes tienen una relación inversa con el precio de las viviendas, lo cual afecta negativamente el valor de venta de estas.

Con el fin de corregir los sesgos ocurridos en la valoración del ruido generado por ferrocarriles, Brons y otros (2003) plantean el establecimiento de un modelo de relación causal para determinar el verdadero costo social y económico ocasionado por los trenes. Las medidas de política destinadas a reducir los costos sociales y económicos se han incorporado en las distintas etapas del modelo causal. Estas medidas pueden subdividirse en dos: la regulación del ruido y las medidas de prevención

16 Moneda de noruega, llamada corona noruega, donde un 1 USD equivale a 6.8111 NOK (tasa de cambio a septiembre de 2004) directa. Por último, sugieren que una forma de probar la eficiencia de su modelo es desarrollar un análisis costo-beneficio.

Por otra parte, Theebe (2004) buscó por medio de la aplicación de regresiones espaciales, y la aplicación del NDI, determinar la clase de relación existente entre los precios de los hogares y los niveles de ruido ocasionados por las estaciones de trenes, cuyo objetivo final era la determinación de herramientas para la toma de decisiones futuras. Dentro de los hallazgos más relevantes expuestos por este trabajo se tiene que a niveles de ruido cercanos a los 65 decibeles causan reducción de un 12\% de los precios de los hogares en Holanda. Sin embargo, cuando las viviendas están situados en zonas con niveles de ruido menores a $40 \mathrm{~dB}$ el precio de las viviendas se incrementa, en promedio, un $6.5 \%$.

De manera alternativa, Nunes, y Travisi (2006) proponen la aplicación de la metodología de experimentos de elección para valorar el ruido. Dentro de su investigación, llevada a cabo en Italia, se pueden resaltar tres aspectos fundamentales. Esta investigación no considera solamente la evaluación económica de la reducción en los niveles de ruido, sino también cómo se consiguen estas reducciones, a través del examen de los instrumentos de política y de tecnología disponibles para tales fines. Por último, el estudio ofrece una contribución original a la literatura de la valoración ya que prueba de manera formal la solidez de las estimaciones econométricas de los experimentos de elección en tres vehículos de pago: (1) Un impuesto especial regional (2) La reasignación de recursos financieros en el presupuesto provincial en el sector del transporte público, y (3) La reasignación de los recursos financieros del presupuesto provincial de la administración y el sector del entretenimiento.

Bellinger (2006) establece una correlación entre los niveles de ruido y la disminución de los precios de las viviendas ubicadas en partes cercanas a la carrilera del tren, al mismo tiempo que demuestra 
el grado de correlación existente entre los niveles de molestia y la disposición a pagar de los habitantes por evitar este ruido. Este autor encuentra que los valores estimados de las propiedades residenciales para Pensilvania (EE. UU.) tienden a disminuir en cerca de 4.800 dólares, un 4.1\% en promedio, por cada 10 dB de la exposición al ruido. El estudio primario se complementó con información recolectada por medio de una encuesta. Similarmente, Clark (2006), por medio de la aplicación del método de precios hedónicos, valora los niveles de ruido generados por los trenes y sus bocinas en las intersecciones viales. Según los resultados de sus análisis, al igual que Bellinger (2006), determina una relación inversa entre los precios de las viviendas y la cercanía a las vías de los trenes.

En la tabla 3 se presenta el resumen de los resultados de los principales estudios revisados de valoración económica de impacto por ruido generado por los trenes y vías ferroviarias con respecto al escenario de valoración propuesto utilizado.

Finalmente, algunos países de la Unión Europea, con el objetivo de facilitar la aplicación de metodologías de valoración económica, han tratado de estandarizar los métodos de cálculo para los diferentes niveles y clases de ruido. Lo anterior facilita la estimación de los efectos acústicos marginales. En este sentido, Andersson y Ogren (2007), en un estudio realizado para Suecia, determinan el costo marginal social de corto plazo según el nivel de ruido ferroviario percibido. Estos costos marginales están asociados a la exposición al ruido generado por el transporte ferroviario de carga y pasajeros. Dicho costo se obtiene a través de la aplicación del método de costo de daño (CD), particularmente, mediante el cálculo de costos de enfermedad ${ }^{17}$.

17 El método de costo de daño tiene como propósito estimar el costo de un recurso asociado con cambios ambientales. Ha sido aplicado para estimar daños materiales, pero en especial para calcular valores económicos de impactos negativos en la salud. En el caso de los costos de enfermedad están compuestos por: 1. Costos médicos y de servicios de salud, 2 . Costos de oportunidad (pérdida de productividad y el costo
El estudio encuentra que los costos marginales sociales estimados son de $0.026,0.099$ y 0.89 euros por $\mathrm{Km}$, para los trenes de cercanías, alta velocidad y de mercancía, respectivamente.

Al evaluar los estudios de valoración del ruido generado por tráfico de trenes, se destaca que los análisis sobre el valor del ruido incluyen de forma implícita las decisiones de política pública guiada a la reducción de los niveles de ruido generados, variable que pocas veces es considerada en los estudios de valoración aplicados para otras fuentes de ruido. Por otra parte, se mantiene alguna regularidad en el uso de las metodologías de precios hedónicos y valoración contingente para el desarrollo de ejercicios de valoración de ruido, aunque se evidencia la presencia de otras metodologías como los experimentos de elección. Finalmente, se concluye que en concordancia con los análisis hechos para las demás fuentes de ruido, los altos niveles de contaminación acústica generan bajas en los precios de las propiedades y viviendas, así como también generan altos niveles de molestia a las poblaciones humanas cercanas a la fuente de ruido.

\subsection{Revisión de estudios de valoración económica del ruido en Latinoamérica}

El problema de la contaminación por ruido es un tema que cobra cada vez mayor relevancia en las agendas ambientales locales de las ciudades latinoamericanas. Su incremento afecta, cada vez más, los entornos urbanos y la calidad de vida de los habitantes de las grandes ciudades. Por tal razón, en la actualidad se han desarrollado en la región algunos estudios en torno a la problemática. No obstante, los estudios de valoración económica del ruido en América Latina y particularmente en Colombia han sido escasos. Aunque la mayoría de los habitantes de las grandes ciudades latinoame-

de oportunidad del ocio del individuo y, 3. La desutilidad, es decir, otros costos económicos y sociales como disconformidad y ansiedad (Anderson y Ogren, 2007, p. 206). 
Tabla 3. Principales estudios revisados de valoración económica de impacto por ruido generado por los trenes y vías ferroviarias

\begin{tabular}{|l|l|l|}
\hline \multicolumn{1}{|c|}{$\begin{array}{c}\text { Estudio } \\
\text { (Metodología de valoración) }\end{array}$} & \multicolumn{1}{|c|}{$\begin{array}{c}\text { Sitio/Descripción del escenario } \\
\text { (Escenario de valoración propuesto) }\end{array}$} & \multicolumn{1}{c|}{ Resultados } \\
\hline $\begin{array}{l}\text { Strand y Vagnes (2001) } \\
\text { PH }\end{array}$ & $\begin{array}{l}\text { Noruega } \\
\text { (Cercanía de los hogares a las vías } \\
\text { férreas) }\end{array}$ & $\begin{array}{l}\text { Incrementos en los precios de las vi- } \\
\text { viendas en 10\% por metro de distancia } \\
\text { de lejanía de las vías. }\end{array}$ \\
\hline $\begin{array}{l}\text { Theebe (2004) } \\
\text { PH }\end{array}$ & $\begin{array}{l}\text { Holanda } \\
\text { (Cercanía de los hogares a las vías } \\
\text { férreas) }\end{array}$ & $\begin{array}{l}\text { Niveles de ruido mayores o iguales a los } \\
65 \mathrm{~dB} \text {. Causan una reducción de precio } \\
\text { de las viviendas en 12\%. }\end{array}$ \\
\hline $\begin{array}{l}\text { Andersson y Ogren (2007) } \\
\text { CD }\end{array}$ & $\begin{array}{l}\text { Suecia } \\
\text { (Niveles de ruido percibidos) }\end{array}$ & $\begin{array}{l}\text { Tasa de depreciación del valor de } \\
\text { las viviendas según niveles de ruido } \\
\text { percibidos 0.026-0.099 y 0.89 euros/ } \\
\text { km, para trenes de cercanías, de alta } \\
\text { velocidad y carga, respectivamente- }\end{array}$ \\
\hline $\begin{array}{l}\text { Bellinger (2006) } \\
\text { PH }\end{array}$ & $\begin{array}{l}\text { Estado Unidos } \\
\text { (Cercanía de los hogares a las vías } \\
\text { férreas) }\end{array}$ & $\begin{array}{l}\text { DAP: 4,800 dólares por incremento de } \\
10 \mathrm{~dB} \text {, en los niveles de ruido. }\end{array}$ \\
\hline
\end{tabular}

Fuente: Correa y Osorio (2010, p. 30)

ricanas padecen las consecuencias del ruido, en pocas ciudades del continente se han emprendido investigaciones rigurosas para determinar, a través de la valoración económica, la pérdida de bienestar asociada a esta relevante externalidad negativa.

En total solo se hallaron estudios de valoración económica del ruido para cuatro ciudades de Latinoamérica: Santiago de Chile (Chile), Buenos Aires (Argentina) y Bogotá y Medellín (Colombia). Al igual que en los estudios de valoración del ruido realizados en otros países como Estados Unidos, España y Países Bajos, en Latinoamérica se han utilizado los métodos de valoración contingente y precios hedónicos como enfoques para determinar la pérdida de bienestar o los beneficios netos asociados a un aumento o disminución de los niveles de ruido. De igual manera, el ruido por tráfico vehicular, ferroviario y aeroportuario es la externalidad que concentra la mayor atención, después de la contaminación atmosférica, en las agendas ambientales locales de las ciudades latinoamericanas.

Así, por ejemplo, Galilea y Ortúzar (2005), llevaron a cabo la estimación de la disposición a pagar de los habitantes de una zona residencial en Santiago de Chile por la disminución de los niveles de ruido. El experimento consideró las variables: el tiempo de viaje al trabajo, el costo del alquiler mensual de la casa, la orientación solar de la vivienda y los niveles de ruido dentro y fuera de ella. Se estimaron modelos Logit multinomial y mixto con base en un marco microeconómico consistente. En este estudio se diseñó una encuesta que tuvo en cuenta la selección de un contexto en el que las familias residentes hubieran estado expuestas a diferentes niveles de ruido. Ahora, a través del uso de un modelo multinomial logit para funciones de utilidad lineales, en el que se corrieron dos modelos MNL-1 ${ }^{18}$ y MNL- $2^{19}$, la disposición a pagar por una disminución en los niveles de ruido fue de US\$23,54 y US\$23,45, respectivamente. Para un modelo logit mixto, en el que se corrieron tres modelos MNL-4, ML-1 y ML-4, la disposición a pagar mensual por la disminución en los niveles de ruido fue de US\$23,68, US\$25,15 y US\$33,98, respectivamente.

18 Modelo Logit Multinomial (MNL 1) que impone homogeneidad de las preferencias de la población.

19 Modelo logit multinomial (MNL 2) que recoge heterogeneidad sistemática en las preferencias por el tiempo de viaje (Amador y González, 2005). 
De otro lado, la Pontificia Universidad Católica de Chile (1999) desarrolló un estudio para conocer los beneficios sociales de la introducción de buses a gas natural comprimido en la región metropolitana de Santiago de Chile. El estudio valoró la disminución del ruido interior y exterior producido por un cambio en la flota de servicio público de diésel a gas natural, a través de la disposición a pagar por la disminución en estos niveles.

Para conocer los niveles de ruido reducido, se utilizó información de estudios anteriores en los que se calculó la mejora acústica por el cambio de buses y el precio de las viviendas de cada uno de los distritos de Santiago y se utilizó un índice de revalorización de las propiedades de $0.4 \%$. De acuerdo con los autores, este dato se extrajo de otros estudios en los que se estimó, por precios hedónicos, el cambio en los precios de la propiedad por cambios en los niveles de ruido. El beneficio social debido a reducciones de ruido interior y exterior por la introducción de buses a gas natural comprimido convencional fue CH\$480.000/bus/año y CH\$1’310.000/bus/año.

Por su parte, Collados (2000) valoró los efectos del ruido urbano en Santiago de Chile. Así, utilizó perfiles de ruido medidos en 180 distritos y datos de población con el fin de estimar el número de habitantes afectados y la fracción de esta población calificada como altamente molesta. Para esto, aplicó un modelo econométrico de precios hedónicos para estimar el costo de depreciación no laboral, y un modelo de pérdidas directas para obtener la reducción de tiempo laboral en Santiago. Para tal fin, se valoraron económicamente las principales actividades que se afectan por el ruido como el descanso y el ocio; también, algunas actividades de tipo funcional, como conducir, ir de compras, el trabajo doméstico y la pérdida de tiempo efectivo de producción.

La valoración de las actividades remuneradas se calculó de acuerdo con la pérdida de horas de trabajo por las interrupciones, la dificultad de las comunicaciones, los errores y la fatiga durante la jornada laboral. Las pérdidas diarias alcanzaron 2,57 millones de dólares, es decir, unos 600 millones de dólares anuales. Asimismo, el valor comercial agregado de las viviendas en los 18 distritos se estima en 58.200 millones de dólares. La depreciación por ruido resultante es de 2.273 millones de dólares, es decir, $6.4 \%$ del valor. Por otra parte, de acuerdo con el autor, el efecto neto que tendría reducir todos los niveles exteriores en $1 \mathrm{~dB}$ en toda la ciudad fue de 528 millones de dólares, es decir, 0.49\% del valor comercial agregado de las viviendas.

Conte (2001) hace una valoración económica de la contaminación por aire y por ruido de la ciudad de Buenos Aires, a través del método de precios hedónicos. Debido a la escasa información para la contaminación por aire y ruido, este autor utiliza como variable proxy el transporte público de pasajeros.

Conte (2001) utiliza una función Box-Cox lineal y estima el modelo econométrico por el método de máxima verosimilitud. Los resultados estadísticos de esta investigación muestran que el precio de los inmuebles y sus características evidencian una relación de forma Log-Log. Las variables que son significativas estadísticamente para explicar los precios de las viviendas fueron: superficie cubierta, antigüedad, cantidad de pisos del edificio, cocheras, y cercanía a una plaza. Por su parte, la variable asociada con el transporte público, que es la variable proxi vinculada a la contaminación del aire y de la ruido, influyó negativamente en los precios, pero con un valor bajo y con una débil significancia al nivel de 15\% (Conte, 2001, p.13).

Por otra parte, Oscanoa (1999), llevó a cabo la valoración económica de los beneficios por la disminución del ruido por tráfico aéreo del aeropuerto El Dorado de la ciudad de Bogotá. Esta valoración se hizo a través de la estimación de una función de precios hedónicos en la que el precio de las viviendas depende de sus atributos. Así, las condiciones ambientales, como lo es el nivel de ruido exterior, 
afectan directamente su precio y el bienestar de quienes habitan en ellas.

De esta manera, el estudio calculó la ganancia de bienestar por la disminución en los niveles de ruido ocasionado por el tráfico aéreo del aeropuerto El Dorado. Las variables explicativas del precio de las viviendas fueron: la suma ponderada de la distribución espacial de los precios de las viviendas, el área total del terreno, el número de alcobas, el tipo de vivienda, el tiempo de acceso a un centro comercial, el tiempo de acceso a un parque y el nivel de ruido y tráfico aéreo. De acuerdo con los resultados, los signos de las variables explicativas fueron los esperados, es decir, la relación entre el precio de la vivienda y los metros cuadrados, y el número de alcobas, fue directa. De igual manera, cuando los niveles de ruido aumentan el precio disminuye, y viceversa.

La disposición a pagar, por $\mathrm{m}^{2}$, por disminución de un decibel en el ruido, cuando se está en niveles de ruido promedio de $70.04 \mathrm{~dB}$, es de $\$ 6.333$ (valores del año 1998). Ahora, la disposición a pagar por $m^{2}$ por disminución de un decibel, cuando se está en niveles de ruido promedio de $55 \mathrm{~dB}$, es de $\$ 8.065$ (valores del año 1998). De otro lado, los beneficios totales por persona por $\mathrm{m}^{2}$ son de $\$ 107.000$ por cada propiedad. Si se toma el área promedio de la propiedad en $93.2 \mathrm{~m}^{2}$, los beneficios ascienden a \$9`970.000. De la estimación y especificación de los precios hedónicos de la vivienda, se puede observar que el precio de esta crece al disminuir el nivel de ruido. Así, la persona está dispuesta a pagar cada vez más por una reducción adicional de esta externalidad.

Finalmente, Correa y Osorio (2010) valoraron económicamente los impactos ambientales asociados al ruido generado por los equipos de aire acondicionado en centrales telefónicas en Medellín, Colombia. Para esto, el estudio en mención utiliza la metodología de VC. En este sentido, se utilizó una encuesta contingente tipo referéndum como herramienta de recolección de información para la realización del ejercicio de valoración. En esta encuesta se incluye, como pregunta principal, la DAA por los efectos negativos generados por el ruido producido por los equipos de aire acondicionado. En este trabajo se utiliza el modelo econométrico de la función Bishop y Herberlein para estimar la DAA de los hogares. Así, se establece el valor económico de la pérdida de bienestar por hogar generada por la percepción del ruido de los equipos de aire acondicionado, el cual corresponde a una DAA media de Col\$19.015/ hogar/ mes por 5 dB(A) y una DAA mediana de Col \$13.541/hogar/mes por $5 \mathrm{~dB}(\mathrm{~A})$.

Posteriormente, los autores plantean la preferencia de la utilización de la mediana sobre la media de la DAA como medida de bienestar más adecuada, pues argumentan que la primera de estas dos medidas de bienestar se ve influida en menor medida por las perturbaciones aleatorias causadas por la posible presencia de observaciones atípicas de la DAA (Hanemmann, 1984). Finalmente, se obtuvo que el valor económico del impacto ambiental negativo anual por cada hogar, medido a partir de la DAA, equivale a Col\$162.498. Correa y Osorio (2010) concluyen en el estudio que la percepción de ruido asociado a los equipos ubicados en las centrales telefónicas se ve fuertemente influenciado por el ruido de fondo existente en los lugares de estudio, en particular del ruido proveniente de flujo vehicular y del ruido generado por los vecinos. Dicha influencia conduce a comportamientos oportunistas, que pueden llegar a acrecentar las expectativas de los individuos por obtener un ingreso extra ante la posibilidad de recibir una compensación monetaria.

\section{CONCLUSIONES}

Uno de los problemas que ha surgido a partir del crecimiento de las actividades económicas y, por supuesto, de las ciudades modernas es el ruido. A medida que crecen las ciudades, aumenta el ruido urbano. En la literatura económica se considera al ruido como un contaminante de bajo costo de 
generación, el cual presenta características particulares que dificultan el establecimiento de su valor económico. En ese sentido, a la luz de los diversos estudios analizados, se puede establecer que los impactos más relevantes del ruido son la incidencia negativa sobre el precio viviendas y las molestias percibidas por los individuos, mediante el sentido del oído. Así, desde el tema del efecto del ruido sobre el precio de las viviendas, los diferentes estudios permiten observar la relación inversa entre el incremento de los niveles de ruido y el precio de las viviendas, lo cual revela, finalmente, una afectación negativa del ruido en el precio de estas, así como DAP positivas de los individuos por reducciones en los niveles de ruido.

Igualmente, se ha expuesto que el ruido, como externalidad negativa, presenta dificultades para ser internalizada. Adicionalmente, hoy en día este problema es de gran interés público debido a la creciente contaminación acústica relacionada con el desproporcionado aumento de las actividades económicas en el entorno mundial. Esta situación ha llevado, inicialmente, a que los gobiernos hayan tomado medidas como la creación de mapas de ruido, especialmente de las zonas urbanas, para mejorar la información referente a la problemática ambiental con el objetivo de proponer acciones de política que busquen minimizar la afectación en el bienestar social.

Así, lo que puede evidenciarse desde las diferentes investigaciones analizadas es que los métodos de valoración económica del medioambiente son importantes para soportar las decisiones de política pública y planeación del territorio que vayan dirigidas a mitigar el impacto negativo de este problema ambiental. De esta forma, mediante la utilización de métodos como el de precios hedónicos y el de valoración contingente se han logrado establecer estimaciones bastante confiables del valor económico del ruido, las cuales han permitido reducir la incertidumbre con respecto a las posibles formas para ejercer un adecuado control de la contami- nación auditiva y sus diferentes afectaciones a la sociedad.

Por su parte, los estudios de valoración económica del ruido en América Latina y, particularmente en Colombia, han sido escasos. Así, aunque la mayoría de la población de las grandes ciudades latinoamericanas padece las consecuencias del ruido, en pocas ciudades del continente se han emprendido investigaciones rigurosas para determinar, a través de la valoración económica, la pérdida de bienestar asociada a esta relevante externalidad negativa.

Finalmente, es importante notar que casi todos los ejercicios de valoración económica del ruido están limitados a los efectos causados por el tráfico terrestre y aéreo. Por lo tanto, es importante desarrollar estudios que permitan la estimación económica de la pérdida de bienestar asociada a diferentes contextos como la exposición a niveles de ruido generados en el trabajo, en los viajes, en la operación de industrias, en el funcionamiento de infraestructura de telecomunicaciones, y generación de electricidad, entre otras fuentes emisoras de ruido.

\section{BIBLIOGRAFÍA}

Amador Morera, Francisco Javier y Gonzáles Marrero, Rosa M. (2005). El valor subjetivo del tiempo de viaje de los estudiantes universitarios cuando las preferencias son heterogeneas. En: Hacienda Pública Española / Revista de Economía Pública. $\mathrm{N}^{\circ}$ 174, p. 25-42.

Andersson, H. and M. Ögren (2007). Noise Charges in Rail Infrastructure: A Pricing Schedule Based on the Marginal Cost Principle. En: Transport Policy. $\mathrm{N}^{\circ} 14$, Vol 3. p. 204-213.

Bateman, Ian J.; Day, Brett H.; Lovett, Andrew A.; Lake, Iain R. (2000). Assigning a Monetary value to Noise reduction benefits; an example from the UK. London, Center for Social and Economic Research on the Global Environment University of Est Anglia And University College, 16p. 
Bateman, Ian; Day, Brett; Lovett, Andrew y Lake, Iain. (2001). The effect of road traffic on residential property values: a literature review and hedonic pricing study. E.S.R.C Economic E Social Research Council. 207 p.

Bellinger, William, K. (2006). The Economic Valuation of Train Horn Noise: A US Case Study. En: Transportation Research, Part D. Transport and Environment. $\mathrm{N}^{\circ} 11$, p. $310-314$

Bishop, Richard y Herbelein, Tomas. (1979). Measuring values of extra-market goods: are indirect measures biased? En: American Journal of Agriculture Economics, Vol. 61, N 5, p. 926-930.

Bluhm, G.; Nordling E. y Berglind N. (2004). Road traffic Noise and annoyance - An increasing Environmental Health problema. En: Noise \& Health. Vol. 6, $N^{\circ} 24$, p. 43-49.

Botton, Kenneth. (2003). The potential of meta-analysis and value transfers as part of airport environmental appraisal. En: Journal of Air Transport management, Vol. 9, N³, p. 167-176.

Brons, Martin; Nijkamp, Peter; Pels, Eric y Rietveld, Piet. (2003) Railroad Noise: economic valuation and policy. En: Transp Research Part D Transport Environment Vol. 8, N 3, p. 169-184.

Caraballo, Javier y Mendieta, Juan. (2005). Economía de la Contaminación y la Degradación Ambiental. Fondo Editorial nuevo tiempo. San Cristóbal Venezuela, $494 \mathrm{p}$.

Carlsson, Frederik; Lampi Elina y Martinsson Peter. (2004). The marginal values of Noise disturbance from air traffic: does the time of the day matter? En: Transportation research. Part D, Transport and environment. Vol. 9, $\mathrm{N}^{\circ}$ 5, p. 373-385.

Clark, David. (2006). Externality Effects of Residential Property Values: The Example of Noise Disamenities. En: Growth and Change. Vol. 37, p. 460-488.

Collados, Eugenio. (2000). Costo social del ruido urbano en Santiago de Chile. En: Tecniacústica. Vol. 31, $\mathrm{N}^{\circ} 2$, p. $1-7$.

Conte, María. (2001). Una primera aproximación a la valuación hedónica de la contaminación en Buenos
Aires, CEMA Working Papers: Serie Documentos de Trabajo, $\mathrm{N}^{\circ} 230$, Universidad del CEMA, 21p.

Correa, Francisco y Osorio, Juan. (2010). Valoración economica de los impactos ambientales asociados a la emision de ruido de las instalaciones de EPM Telecomunicaciones S.A. E.S.P. Mimeo. Informe Final proceso de contratación 071569. Medellín, Colombia, 220p.

Correa, Francisco, (2007), Evaluación económica de impactos ambientales: una guía metodológica para la determinación de la tasa social de descuento, Medellín, Sello Editorial Universidad de Medellín, 113 p.Correa, Francisco. (2007). Evaluación económica de impactos ambientales: una guía metodológica para la determinación de la tasa social de descuento. Medellín. Sello Editorial Universidad de Medellín, $113 p$.

Faburel, Guillaume. (2001) Le bruit des avions: évaluation du coût social. Entre aéroport et territoires: Ed. Presses de l'Ecole Nationale des Ponts et Chaussées, Paris, 350p.

Flowerdew, A.D.J. (1972). The cost of airtport Noise. En: Journal of the Royal Statitical Society. Vol. 21, $\mathrm{N}^{\circ}$ 1, p. 31-46.

Galilea, Patricia y Ortuzar, Juan. (2005). Valuing Noise level reductions in a residential location context. En: Transportation Research, Part D. Transport and Environmental. Vol. 10, N 4. p. 305-322.

Garrod, G.D. y Willis K.G. (1992). Valuing goods characteristics: An application of the hedonic Price method to environmental attributes. En: Journal of Environmental Management, $\mathrm{N}^{\circ}$ 34, Vol. 1, p. 59-76.

Hanemman, Michael.W. (1984). Welfare evaluations in contingent valuation experiments with discrete responses data. En: American Journal of Agriculture Economic. Vol. 71, N 4, p. 1057-1061.

Haro, Alma y Taddei, Cristina. (2010). Valoración ambiental: aportaciones, alcances y limitaciones. En: Problemas del desarrollo. Vol 41, No 160, p. 209-221.

Ising, Hartmut y Ising, Martin. (2002). Chronic Cortisol Increases in the First Half of the Night Caused by 
road traffic Noise. En: Noise and Health. Vol. 4, $\mathrm{N}^{\circ}$ 16, p. 13-21.

Jasper, Dekker y Straaten, Willemijn van der (2008). Monetary valuation of aircraft Noise: A hedonic analysis around Amsterdam airport. En: Journal of Ecological Economics, $\mathrm{N}^{\circ} 68, \mathrm{~N}^{\circ} 11$, p. 2850-2858.

Lamarque, J. (1975). Le droit contre le bruit. LGDJ, Paris, France. 380p.

Lambert, J. Poisson, F. Champlovier p. (2001). "Valuing benefits of road traffic Noise abatement program: A contingent valuation study". INRETS-LTE, Bron, France. Paper presented at the $17^{\text {th }}$ international congress on acoustics, $2 \mathrm{p}$.

López, Isabel y Herranz, Karmele (1991). Ruido de tráfico e interferencia en el sueño. Psicología ambiental: intervención y evaluación del entorno. Arquetipo, Sevilla, p. 309-316.

Marmolejo D. y Frizzera, A. (2008) Cuanto estamos dispuestos a pagar por el silencio? Un análisis contingente para la ciudad de Barcelona. ACE: Arquitectura, Ciudad y Entorno, Any III, Nº 7, p. 21-40.

Marmolejo, Carlos (2008), La incidencia de la percepción del ruido ambiental sobre la formación espacial de los valores residenciales: un análisis para Barcelona. En: Revista de la Construcción, Vol. 7, $\mathrm{N}^{\circ}$ 1, p. 4-19.

Martínez, Avelino (2005). Ruido por tráfico urbano: conceptos, medidas descriptivas y valoración económica. En: Revista de economía y administración, Vol. 2, $N^{\circ} 1$, p. 1-49.

Meister, Edward y Donatelle, Rebecca. (2000). The impact of commercial aircraft Noise on Human Health: A neighborhood study in metropolitan Minnesota. En: Journal of Environmental Health, Vol. 63, p. 8-15.

Mendieta, Juan; Uribe, Eduardo; Rueda, Haider y Carriazo, Fernando (2003). Introducción a la valoración ambiental, y estudios de caso. Bogotá D.C. Universidad de los Andes. 227p.

Miedema, Henk y Vos, Henk (1998). Exposure response relationships for transportation Noise. En: The Journal of the Acoustical Society American. Vol. 104. $N^{\circ}$ 6, p. 3.432-3.445.
Navrud, Stales. (2000). Valuation Techniques and Benefit Transfer Methods: Strengths, Weaknesses and Policy Utility. Valuation Rural amenities, 180p.

Navrud, Stales (2002). The State of art on economic valuation of Noise. (Final report to European Commission DG Environment). Department o Economics and Social Sciences, Agricultural University of Norway, 68p.

Nunes, Paulo y Travisi, Chiara (2006). Comparing Tax and Tax Reallocations Payments in Financing Rail Noise Abatement Programs: Results from a CE valuation study in Italy. Fondazione Eni Enrico Mattei. Venecia, Italia, 33p.

Nunes, Paulo y Travisi, Chiara (2008). Comparing tax an tax reallocation payments in financing rail Noise abatement programmes: Results from a stated choice valuation study in Italy. En: Enviromental and Resource Economics,Vol. 43, N 4, p. 1573-1502.

OMS -Organización Mundial de la Salud- (1999). Guidelines for Community Noise, Ginebra, p. 250-275.

Ortega, Domínguez (2002). Ruido: efectos sobre el sueño. Jornadas internacionales: contaminación acústica en las ciudades. Madrid, España.

Oscanoa, Héctor (1999). Valoración económica de los beneficios por la disminución del nivel de ruido por tráfico aéreo en Santa Fe de Bogotá. Tesis de MSc., Universidad de los Andes, 55p.

Osorio, Juan David (2006). El método de transferencia de beneficios para la valoracion enconomica de servicios ambientales: estado del arte y aplicaciones. En: Semestre Económico. Vol. 9, № 18, p. 107-124.

Osorio, Juan David y Correa, Francisco. (2009). Un análisis de la aplicación empírica del método de valoración contingente. En: Semestre Económico. Vol. 12, $\mathrm{N}^{\circ} 25$, p. 11-30.

Paz, Elaine y Zannin, Paulo (2008). Perception of Noise in suburban and urban areas. En: The Journal of The Acoustical Society Of America. Vol. 123, No 8, p. 5.789-5.793.

Pearce, D. And Turner (1990). "Economía de los Recursos Naturales y Ambientales". Colegio de Economistas de Madrid. Celeste Editores. España, 448p. 
Francisco Correa Restrepo - Juan David Osorio Múnera - Bernardo Patiño Valencia

Pommerehne, W. (1988). Measuring environmental benefits: a comparison of hedonic techniques and contingent valuation. Welfare and Efficiency in Public Economics, London, 424p.

Pontificia Universidad Catolica de Chile (1999). Estudio de seguimiento del plan piloto de utilización de combustibles gaseosos en buses de la Región Metropolitana. En: Evaluación económica. Vol. 2, 37p.

Poon, Larry (1978). Railway Externalities and Residential Property Prices. En: Journal of Land Economics. Vol. 54, N² 2, p. 218-257.

Redman, Milton. (1975). Noise and Prices. Oxford University Press. En: Journal of Behavioral Economics, Vol. 5, p. 195-198.

Regecove, V. y Rellerova, E. (1995). Effects of urban Noise pollution on blood pressure and heart rate in preschool children. En: JHypertens. Vol. 13, $\mathrm{N}^{\circ}$ 4, p. $405-412$.

Rosenberger, R, Loomis, J. (2003). Benefit Transfer, Chapter 12, A Primer on Nonmarket Valuation, Editado por Patricia A Champ y Kevin J Boyle, New York, Thomas C Brown-Business \& Economics, 576p.

Salazar, Salvador. (2004). Tráfico rodado y efectos externos: Valoración económica del ruido. En: Ekonomiaz: Revista vasca de economía, N 57, p. 46-67.

Santford, Fidell; Schultz, Theodore y Green, David M. (1988). A theorical interpretation of the prevalence rate of Noise induced annoyance in residential populations. En: Journal of the acoustical Society of America, Vol. 84, N 6, p. 2109 - 2112.

Sanz, J. M. (1987) El ruido. MOPU. Unidades Temáticas Ambientales de la Dirección General del Medio Ambiente. Madrid, España, 113p.

Schipper, Y.; Nijkamp, p. y Rietveld, p. (1998). Why do aircraft Noise value estimates differ? A metaanalysis. En: Journal Air Transport Management. Vol. 4, $N^{\circ}$ 2, p. 117-124.

Soguel, N. (1994). Evaluation monetaire des atteinties a l'environment: Une étude hedoniste et contingente sur l'impact des transports, Imprimerie de L'evolve SA Neuchatel, 25p.
Stanley, T. D. (2001). Wheat from Chaff: Meta-Analysis as Qualitative Literature Review. En: Journal of Economic Perspectives, Vol. 15, N 3, p. 131-150.

Strand, Jon y Vagnes, Mette (2001). The relationship between property values and railroad proximity: A study based on hedonic prices and real estate brokers appraisals. En: Transportation, Vol. 28, $\mathrm{N}^{\circ}$ 2, p. 137-156.

Theebe, Marcel (2004). Planes, Trains, and Automobiles: The Impact of Traffic Noise on House Prices. En: The Journal of Real Estate Finance and Economics, Vol. 28, p. 209-234.

Thune-Larsen, H (1995). Flystøyavgifter basert på betalingsvillighet. Oslo, Transportøkonomisk institutt, TØI-rapport 289/1995, 35p.

Uribe, Eduardo y Rueda, Jaime (2003). Valoración económica de una mejora en la seguridad y la Conservación del Parque Nacional Chingaza. Informe Final presentado a la empresa de acueducto y alcantarillado de Bogotá. Universidad de los Andes. Bogota, Colombia.

Vainio, Mattio. (1995). Traffic Noise and air pollution: Valuation of Externalities with the hedonic price and contingent valuation methods, PhD Thesis, School of Economics and Business Administration, Helsinki.

Vainio, Mattio. (2001). Comparison of Hedonic Prices and Contingent Valuation Methods in Urban Traffic Noise Context. Paper presented at. International Congress and Exhibition on Noise Control Engineering, The Hague, 30p.

Van Praaq, Bernard. \& Baarsma, Barbara. (2001). Using Happiness Surveys to Value Intangibles: The Case of Airport Noise. En: The Economic Journal. N ${ }^{\circ} 115$, p. 224-246.

Walters, A. A. (1975). Noise and Prices. Oxford University Press, London, 147p.

Wilhelmsson, Mats. (2000). The Impact of Traffic Noise on the Values of Single-Family Houses. En: Journal of Environmental Planning and Management, Vol. 43, $\mathrm{N}^{\circ}$ 6, p. 779-815. 
